\title{
Prevalência da automedicação na população urbana portuguesa
}

\author{
Zilda Mendes ${ }^{1 *}$, Ana Paula Martinss ${ }^{1}$, Ana da Costa Miranda ${ }^{1}$, Maria Augusta Soares ${ }^{2}$, \\ Ana Patrícia Ferreira', Ana Nogueira²
}

\begin{abstract}
${ }^{1}$ Centro de Estudos de Farmacoepidemiologia da Associação Nacional das Farmácias, Portugal, ${ }^{2}$ Centro de Informação do Medicamento da Associação Nacional das Farmácias, Portugal
\end{abstract}

*Correspondência:

Z. Mendes

Centro de Estudos de

Farmacoepidemiologia

Associação Nacional das Farmácias

Rua Marechal Saldanha $\mathrm{n}^{\circ} 1$

1249-069 - Lisboa - Portugal

E-mail: zilda.mendes@anf.pt
Este estudo transversal teve como objetivo principal determinar a prevalência da automedicação em meio urbano. Decorreu nas cidades de Lisboa e Porto, respectivamente em 11 e 15 farmácias, no período de Novembro de 1995 a Setembro de 1996. A recolha de informação dividiu-se em 6 fases de 10 dias cada. A prevalência da automedicação encontrada foi de 26,2\%. Encontraram-se diferenças estatisticamente significativas no que respeita às prevalências da automedicação por sexo, idade, nível educacional e atividade profissional. Em 50\% dos casos, os voluntários que adquiriram medicamentos em automedicação fizeram-no sob aconselhamento do farmacêutico. Os grupos terapêuticos mais utilizados em automedicação foram as preparações nasais e sistêmicas para a constipação e tosse $(23,0 \%)$ e os analgésicos (13,6\%). Analisando cada um dos grupos terapêuticos individualmente foram encontradas diferenças estatisticamente significativas relativamente ao perfil do utilizador por sexo e grupo etário, de acordo com a variável automedicação.

\section{INTRODUÇÃO}

A automedicação é um fenômeno freqüente nos auto-cuidados em saúde, desde há muito utilizado e cuja ocorrência e distribuição estão, naturalmente, relacionados com a organização do sistema de saúde de cada país. O nível de informação e o papel dos médicos e farmacêuticos assume particular importância no acompanhamento dos doentes em automedicação. Em 1997, foi produzido pelas instituições ligadas aos farmacêuticos, médicos e indústria farmacêutica, em nível Europeu (Common Position State Statement, 2 de Outubro de 1997), um documento que identifica como situações passíveis de automedicação a constipação, gripe, tosse, dor de garganta, rinite alérgica, feridas na cavidade oral, indigestão, obstipação, vômitos, diarréia, hemorróidas, queimadura solar, verrugas, dores moderadas (cabeça ou muscular) e alguns problemas de pele. O período para o qual se considera a automedicação como adequada não deve exceder 3-7 dias. Deve ser dedicada especial atenção às grávidas, mulheres nutrizes, bebês e crianças.

Na Bélgica, um estudo sobre automedicação numa amostra de 1000 doentes demonstrou que $38 \%$ recorreram à automedicação durante um período de cerca de 3 meses antes de decidirem consultar o médico (Segbrin, 1993).

Um artigo publicado no British Medical Journal por 
Blenkinsopp e Bradley (1996), revelou que 9 em cada 10 indivíduos tiveram nas duas semanas anteriores ao estudo um problema de saúde, sendo que 3 em cada 4 indivíduos recorreram à automedicação. Os grupos terapêuticos mais utilizados foram os analgésicos $(16,7 \%)$, as preparações dermatológicas (11,3\%), os medicamentos para a constipação e tosse $(12,8 \%)$, as preparações para a orofaringe $(5,7 \%)$ e os medicamentos utilizados em perturbações digestivas $(5,8 \%)$.

No Canadá, em estudo realizado por Segal (1990), numa amostra representativa de alojamentos em Winnipeg e Toronto, verificou-se que os medicamentos mais utilizados eram os analgésicos $(92,9 \%)$, medicamentos para a tosse e constipação $(81,6 \%)$ e vitaminas $(72,1 \%)$.

\section{PARTICIPANTES E MÉTODOS}

O modelo de estudo utilizado foi o descritivo transversal. Foram convidadas a participar no estudo 100 farmácias escolhidas aleatoriamente (60 da área de Lisboa e 40 do Porto). Destas, 11 farmácias do distrito de Lisboa e 15 farmácias do distrito do Porto mostraram-se disponíveis para participar no estudo. Os utentes elegíveis foram selecionados um em cada hora de funcionamento da farmácia, desde que residissem nos distritos de Lisboa e Porto, falassem português e fossem adquirir medicamentos para si ou para outrem menor de 20 anos, incapacitado ou idoso.

A informação foi recolhida através de um questionário com cerca de 15 perguntas, mediante entrevista direta feita pelo farmacêutico, o qual teve formação específica durante dois dias. $\mathrm{O}$ estudo foi efetuado em 6 períodos, contemplando os 4 trimestres do ano, de novembro de 1995 a setembro de 1996, tendo-se previamente realizado um ensaio piloto em outubro de 1995 em duas farmácias de Lisboa e duas, do Porto, com o objetivo de testar a compreensão do questionário e averiguar a exeqübilidade do critério de seleção dos voluntários. O questionário continha variáveis de caracterização sócio-demográfica (sexo, idade, nível de escolaridade e situação perante a profissão), bem como, variáveis relacionadas com as preferências dos voluntários no acesso aos serviços de saúde (nomeadamente, a quem se dirige quando tem um problema de saúde ligeiro e quando tem um problema de saúde grave). Era igualmente efetuado o registo de medicação dispensada, bem como se trazia receita médica e quem lhe tinha aconselhado a tomar a medicação.

Neste estudo, o voluntários estava em situação de automedicação se adquiriu pelo menos um medicamento sem ser por indicação médica.

Os medicamentos foram agrupados de acordo com a classificação Anatómica Terapêutica e Química da OMS (ATC).

Nos testes de hipóteses foi utilizado um nível de significância de $5 \%$, tendo sido realizados os seguintes: Teste de $\mathrm{c}^{2}$ para comparação de proporções, teste t-student ou Mann-Whitney para comparação de médias em duas amostras independentes, e ANOVA ou teste de Kruskal Wallis para a comparação de médias em mais do que duas amostras independentes.

\section{RESULTADOS}

O número total de entrevistas em análise foi de 4.135. A prevalência global da automedicação encontrada foi de 26,2\% (IC 95\% 24,9\%-27,5\%). A amostra de voluntários destas farmácias era composta majoritariamente do sexo feminino (68\%), sendo a idade média encontrada de 46 anos (mínimo $=0$ e máximo $=95$ anos). A maioria dos voluntários da amostra tinha o ensino primário $(38,0 \%), 7,5 \%$ não sabiam ler nem escrever, $13,4 \%$ tinha o ensino preparatório, $26,5 \%$ tinha o ensino secundário e $14,5 \%$ tinham formação superior. Apenas uma parte muito reduzida $(0,1 \%)$ referiu não saber o nível de escolaridade. Segundo a atividade profissional, verificou-se que $45,4 \%$ eram trabalhadores por conta de outrem, $10,3 \%$ por conta própria, $24,1 \%$ reformados, $8,1 \%$ domésticas, $7,5 \%$ estudantes e $4,6 \%$ desempregados. Perante uma situação ligeira de saúde, $27,6 \%$ dos indivíduos referiram recorrer ao farmacêutico, enquanto que, perante um problema grave de saúde cerca de $71 \%$ dos entrevistados referiu que recorriam a instituições públicas.

As prevalências específicas da automedicação, encontradas em cada uma das variáveis em análise constam do gráfico 1 .

A prevalência da automedicação foi mais elevada no sexo masculino $(28,4 \%)$, do que no sexo feminino $(25,2 \%)$. A diferença é estatisticamente significativa $(\mathrm{p}=0,031)$. A prevalência da automedicação foi mais elevada entre os 10 e os 49 anos de idade - cerca de $31 \%$, decrescendo, substancialmente no grupo etário seguinte $(\mathrm{p}<0,001)$, passando a $19,3 \%$ nos indivíduos com idades superiores a 69 anos. No grupo etário dos mais jovens $<10$ anos - a prevalência da automedicação foi de $12,6 \%$. A prevalência da automedicação variou entre os $32,5 \%$ para os utentes que tinham o ensino superior e os $22,5 \%$ para os que tinham menos do que o ensino preparatório. No grupo dos trabalhadores ou estudantes, a prevalência da automedicação foi superior (30,9\%) ao grupo dos não trabalhadores $(19,1 \%)$. Esta diferença é considerada estatisticamente significativa $(\mathrm{p}<0,001)$.

Quando questionados sobre o que faz habitualmente quando tem um problema ligeiro de saúde, os que recor- 


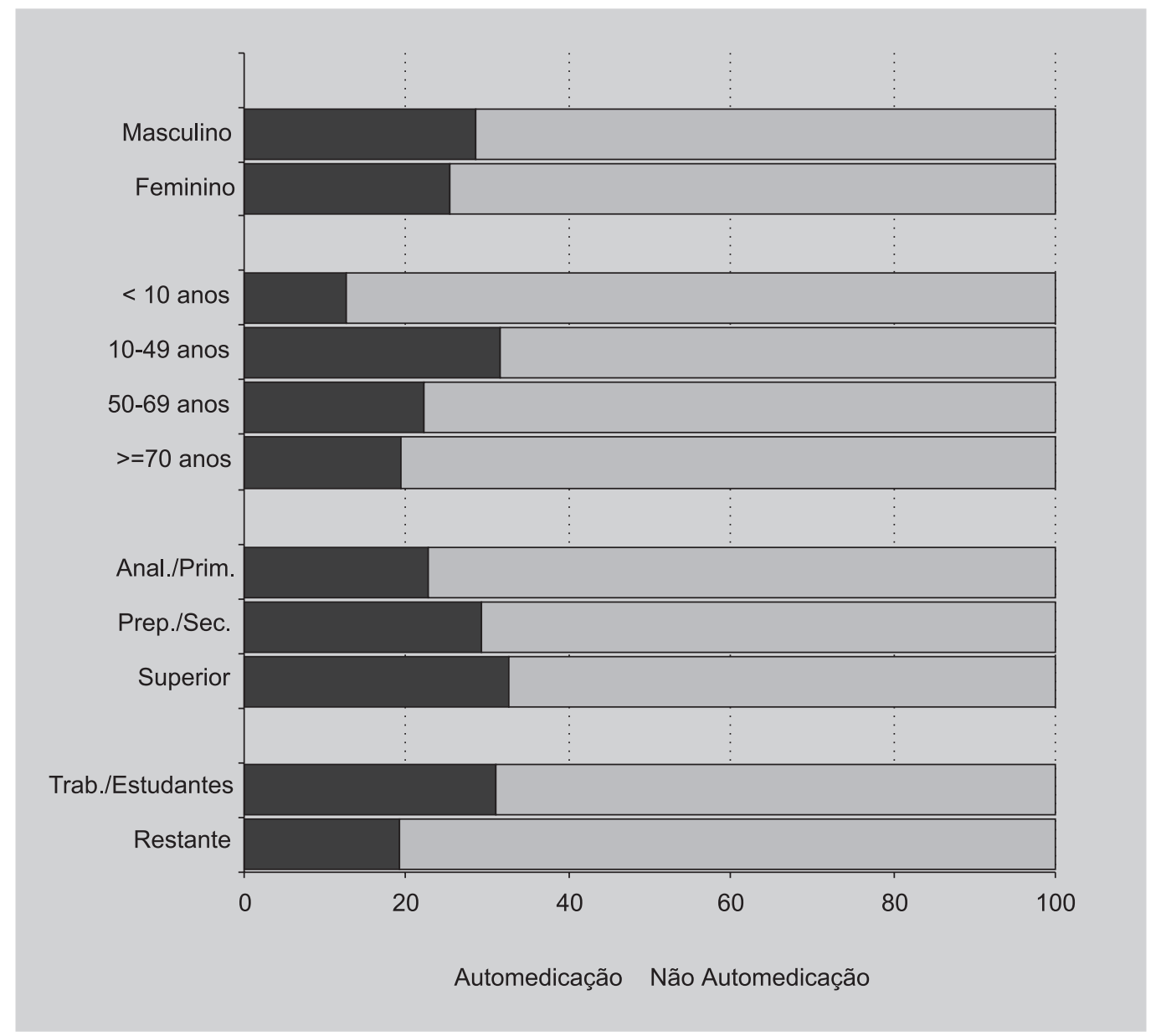

GRÁFICO 1 - Prevalência da automedicação segundo as variáveis de caracterização sócio-demográfica.

rem ao farmacêutico têm maior prevalência de automedicação $(42,8 \%)$ quando comparados com os que recorrem a outros profissionais de saúde. Relativamente ao que fazem quando estão perante um problema de saúde grave, constatou-se que a mais elevada prevalência de automedicação $(27,3 \%)$ se registou nos que referiram recorrer habitualmente a instituições públicas de saúde, quando comparada com os que referiram recorrer a instituições privadas $(23,5 \%)$. Esta diferença também é estatisticamente significativa $(\mathrm{p}=0,012)$.

Dos 8.055 medicamentos adquiridos, durante este estudo, 1.341 foram dispensados em situação de automedicação. Destes 1.341, 670 foram dispensados com aconselhamento farmacêutico $(50,0 \%), 30,1 \%$ foram solicitados ao farmacêutico por iniciativa do voluntário, $18,3 \%$ por sugestão de familiares ou amigos e $1,4 \%$ por indicação do enfermeiro. Para análise detalhada dos grupos terapêuticos foram calculados dois indicadores: (1) prevalência da automedicação por grupo terapêutico (relação entre o $\mathrm{n} .^{\circ}$ de medicamentos adquiridos em autome- dicação de um determinado grupo e o n. ${ }^{\circ}$ total de medicamentos adquiridos desse grupo) e (2) Automedicação proporcional (relação entre o $\mathrm{n}^{\circ}$. de medicamentos adquiridos em automedicação de um grupo terapêutico e o n. ${ }^{\circ}$ total de medicamentos adquiridos em automedicação). Os resultados obtidos encontram-se na Tabela I, apenas para os grupos terapêuticos que apresentaram maiores percentagens para ambos os indicadores.

O grupo mais utilizado em automedicação foi o das preparações nasais e sistêmicas para a gripe e tosse em $23,0 \%$ do total de situações de automedicação (neste grupo foram incluídos os medicamentos pertencentes ao grupo R05 da classificação ATC, associações medicamentosas contendo ácido acetilsalicílico e/ou paracetamol e cafeína e/ou codeína e óleos essenciais e/ou vitaminas, bem como, as associações medicamentosas de antihistamínicos com vasoconstritores locais ou sistêmicos com ou sem analgésico e antipirético). Em segundo lugar, com $16,2 \%$ do total de medicamentos adquiridos em automedicação, encontram-se os análgésicos, essencial- 
TABELA I - Prevalência da automedicação por grupo terapêutico e automedicação proporcional

\begin{tabular}{lcccc}
\hline Grupo terapêutico & Automedicação Não automedicação & $\begin{array}{c}\text { Prevalência } \\
\text { específica (\%) }\end{array}$ & $\begin{array}{c}\text { Automedicação } \\
\text { proporcional (\%) }\end{array}$ \\
\hline Preparações para a orogafinge & 60 & 15 & 80,0 & 4,5 \\
Preparações nasais e sistêmicas & 308 & 226 & 57,7 & 23,0 \\
$\quad$ para a gripe e tosse & & & & \\
Preparações estomatológicas & 62 & 56 & 52,5 & 4,6 \\
Laxantes & 60 & 80 & 42,9 & 4,5 \\
Analgésicos & 217 & 438 & 33,1 & 16,2 \\
Preparações dermatológicas & 153 & 334 & 31,4 & 11,4 \\
Vitaminas & 104 & 383 & 21,4 & 7,8 \\
\hline
\end{tabular}

mente o ácido acetilsalicílico, o paracetamol, a associação do ácido acetilsalicílico com vitamina C. Quanto a preparações dermatológicas, que representaram $11,4 \%$ dos medicamentos adquiridos em automedicação, estas foram, essencialmente, a iodopovidona, o dimetindeno e a bacitracina. As vitaminas, suplementos minerais e outros produtos do trato alimentar e metabolismo $(7,8 \%)$ foram o quarto grupo de medicamentos mais adquiridos em automedicação nesta amostra.

Analisando as prevalências da automedicação por grupo terapêutico verificou-se que a mais elevada era para as preparações para a orofaringe $(80,0 \%)$, predominando a tirotricina, a associação de tirotricina com o cloreto de cetilpiridínio e o cloreto de oxibuprocaína e a clorexidina. Cinquenta e três por cento dos utilizadores deste grupo eram do sexo feminino e tinham idade mediana de 41 anos. Não se encontraram diferenças, estatisticamente significativas, na distribuição por sexo e idades entre os voluntários que se automedicaram e os que não se automedicaram. No grupo das preparações nasais e sistêmicas para a gripe e tosse verificou-se que $57,7 \%$ dos medicamentos deste grupo foram adquiridos em situação de automedicação, sendo mais freqüente a utilização da associação de ácido acetilsalicílico com codeína e cafeína. Analisando por sexo e idade constatou-se que $69,3 \%$ eram do sexo feminino com idade mediana de 45 anos, sendo os voluntários que não se automedicaram mais jovens $(p=0,012)$ do que os que se automedicaram. Analisando as preparações estomatológicas, $52,5 \%$ foram dispensadas em situação de automedicação. As mais utilizadas foram a preparações que continham na sua composição a hexetidina, a benzidamina e o cloreto de zinco. Cerca de $50 \%$ dos voluntários tinham pelo menos 41 anos e $61,7 \%$ eram do sexo feminino sendo, bastante mais jovens os indivíduos que não se automedicaram $(\mathrm{p}=0,023$ idade mediana de 34 anos versus 47 anos). Vale salientar, ainda, a elevada prevalência de utilização dos laxantes, dos analgésicos e das preparações dermatológicas em situação de automedicação, nas proporções de 42,9\%,33,1\% e 31,4\%, respectivamente.

\section{DISCUSSÃO E CONCLUSÕES}

Este estudo contribuiu para melhor conhecimento do fenômeno da automedicação na população urbana portuguesa. As farmácias portuguesas evidenciaram ser um local privilegiado para a recolha de informação epidemiológica, tendo-se verificado taxa de adesão bastante aceitável $(26,2 \%)$.

Relativamente a um potencial viés de seleção dos voluntários por parte do farmacêutico, durante o período de formação, estes foram informados de que deveriam selecionar o primeiro voluntário de cada hora. No entanto, por questões de operacionalidade após o ensaio piloto, o critério foi alterado passando o farmacêutico a selecionar um voluntário por hora (independentemente das suas características sócio-demográficas). Por outro lado, analisandose as características dos voluntários que responderam ao inquérito por farmácia, não foram encontradas diferenças que possam explicar seleção não aleatória dos indivíduos.

A prevalência da automedicação encontrada foi de $26,2 \%$ em população essencialmente urbana. Atualmente, o Centro de Estudos de Farmacoepidemiologia da Associação Nacional das Farmácias está desenvolvendo estudo semelhante em população rural, uma vez que, neste tipo de população o fenômeno da automedicação poderá, eventualmente, ser diferente.

Os homens apresentaram prevalência de automedicação maior do que as mulheres. Tal fato não é muito usual, em outros estudos realizados na Europa. Apenas encontramos resultado semelhante em um estudo realizado no Brasil (Castro et al., 2000) 
O fenômeno da automedicação parece não se distribuir igualmente em todos os grupos etários, podendo-se, no entanto, referir que eventualmente existirá maior preocupação em levar as crianças ao médico, uma vez que nas crianças a prevalência da automedicação encontrada foi menor. Há, no entanto, que atentar para o reduzido número de voluntários até aos 10 anos - apenas 45 doentes.

Tendo em atenção os diferentes níveis educacionais e a situação perante o trabalho, a proporção de voluntários em situação de automedicação revelou ser diferente. $\mathrm{O}$ estudo revelou proporção mais elevada de automedicação nos indivíduos com formação universitária e nos que trabalhavam ou eram estudantes.

Quanto têm um problema ligeiro de saúde mais de $1 / 4$ dos voluntários referiram preferir recorrer ao farmacêutico, enquanto que num problema grave de saúde preferem recorrer a instituições públicas. Neste último caso, a prevalência da automedicação é mais elevada provavelmente devido ao fato da menor acessibilidade aos cuidados de saúde por parte das Instituições Públicas.

Neste estudo, cerca de 50,0\% dos medicamentos adquiridos automedicação foram aconselhados pelo farmacêutico.

Dentre o total de medicamentos utilizados em automedicação, os mais frequentemente adquiridos foram as preparações nasais e sistêmicas para a gripe e tosse $(23,0 \%)$ e os análgésicos $(16,2 \%)$.

Analisando em detalhe cada um dos grupos terapêuticos verificou-se que a prevalência de automedicação específica em cada grupo terapêutico foi mais elevada para as preparações orofaríngeas e para as preparações nasais e sistêmicas para a gripe e tosse. Assim, do total das preparações orofaríngeas dispensadas $80,0 \%$ foram adquiridas em automedicação. Já no total dos medicamentos dispensados do grupo das as preparações nasais e sistêmicas para a gripe e tosse, $57,7 \%$ foram adquiridas em automedicação. Analisando o perfil do utilizador destes medicamentos, quanto se encontraram diferenças estatisticamente significativas, estas diziam respeito à distribuição das idades, isto é, os indivíduos mais jovens teriam menor probabilidade de se automedicarem.

\section{ABSTRACT \\ Self-medication prevalence in a Portuguese Urban Area}

To determine the prevalence of self-medication in a Portuguese urban population, a cross-sectional study was carried out in Lisbon (11 pharmacies) and Oporto (15 pharmacies). Data collection took place between November 1995 and September 1996, during 6 periods of 10 days each. The overall prevalence of self-medication found was 26,2\%. Statistical significant differences were found between self-medication prevalence according to sex, age, educational level and professional status. Fifty percent of the individuals who have used drugs in selfmedication did so with the pharmacist advice. The most frequent therapeutic groups used in self-medication were cough and cold preparations (23\%) and analgesics $(13,6 \%)$. Analysing each group separately, statistical significant differences for sex and age were found according to the variable self-medication.

\section{UNITERMS: Self-Medication. Pharmacies.}

\section{AGRADECIMENTOS}

A todos os farmacêuticos participantes neste estudo. Aos Drs. Marinho Falcão, epidemiologista, e Dr. João Silveira, farmacêutico, pelo apoio e sugestões durante a realização deste estudo.

\section{FINANCIAMENTO}

Este estudo foi integralmente financiado pela Associação Nacional das Farmácias.

\section{REFERÊNCIAS BIBLIOGRAFICAS}

SEGBIN, M. Medicaments et traitements "Le guide des médiments pour le consommateur". Ann. Pharm. Belg., v.12, p. 4-5.1993.

BLENKINSOPP, A.; BRADLEY, C. Over the counter drugs: patients, society and the increase in self-medication. $\mathrm{Br}$. Med. J., v. 312, p.629-632, 1996.

CASTRO, L. Incluir os demais autores Algumas características da prática da automedicação em Campo Grande. Mato Grosso do Sul. Rev. Saúde Farmacol., v. 2, p.4-10, 2000.

SEGAL, A. Community Survey of Self-medication Activities. Med. Care, v. 28, p.3010-3310, 1990.

Recebido para publicação em 08 de julho de 2002. 